\title{
Probiotics improve insulin resistance status in an experimental model of Alzheimer's disease
}

\author{
Somayeh Athari Nik Azm ${ }^{1}$, Abolghassem Djazayeri ${ }^{1}$, Majid Safa ${ }^{2}$, Kian Azami ${ }^{3}$, Mahmoud Djalali ${ }^{4}$, \\ Mohammad Sharifzadeh ${ }^{5 *}$, Mohammadreza Vafa6*
}

\begin{abstract}
Background: Nowadays, Alzheimer's disease (AD) is considered as Type 3 diabetes in which insulin resistance is the common cause of both diseases. Disruption of insulin signaling cascade and insulin resistance can induce AD; and central insulin resistance causes systemic alterations in serum insulin, FBS levels, and lipid profile. Studies have shown that probiotics (Lactobacillus and Bifidobacterium species) can be used as a nutritional approach to improve these metabolic changes. We assessed the probiotic effect (4 species of Lactobacillus and Bifidobacterium) on insulin resistance biomarkers in an experimental model of AD.

Methods: A total of 60 rats were divided into 5 groups: (1) a control group without surgical and dietary intervention; (2) a controlprobiotics group receiving probiotics for 8 weeks, but not receiving any surgical intervention; (3) a group receiving a sham operation in which PBS was injected intrahippocampus but without dietary intervention; (4) an Alzheimer group for which Amyloid- $\beta$ (Aß) 1 42 was injected intrahippocampus but without dietary intervention; (5) and an Alzheimer-probiotics group for which Aß1-42 was injected intrahippocampus and given $2 \mathrm{~g}$ probiotics for 8 weeks. The FBS levels and lipid profile were measured by a calorimetric method, insulin levels were detected by an ELISA kit, and HOMA-IR was calculated using a formula. ANOVA (one way analysis of variance followed by Bonferroni comparisons post hoc) was used to compare all the variables between groups.

Results: Serum glucose, insulin levels, and HOMA-IR index increased in the Alzheimer group compared to the control ( $p<0.001$ ), while probiotics decreased only insulin level and HOMA-IR index in AP group compared to Alzheimer group ( $\mathrm{p}<0.001)$. Also, TG levels increased in the Alzheimer group $(\mathrm{p}<0.001)$, but no significant difference was detected between Alzheimer and Alzheimerprobiotics group.
\end{abstract}

Conclusion: It seems that probiotics play an effective role in controlling glycemic status of Alzheimer's disease.

Keywords: Alzheimer Disease, Lactobacillus, Bifidobacterium, Insulin, HOMA

Copyright@ Iran University of Medical Sciences

Cite this article as: Athari Nik Azm S, Djazayeri A, Safa M, Azami K, Djalali M, Sharifzadeh M, Vafa M. Probiotics improve insulin resistance status in an experimental model of Alzheimer's disease. Med J Islam Repub Iran. 2017 (18 Dec);31:103. https://doi.org/10.14196/mjiri.31.103

\section{Introduction}

Alzheimer's disease (AD), as a neurodegenerative disease, causes impaired cognition function and memory loss (Tillisch et al. 2013). Nowadays, the number of patients with $\mathrm{AD}$ is estimated to be 36 million worldwide, and it is estimated that the number will be quadrupled in 2050 (Ríos et al. 2014, Saxena et al. 2011). Although less than $5 \%$ of $\mathrm{AD}$ cases are the inherited familial type, the sporadic type (late-onset) is the most common type of $\mathrm{AD}$,

Corresponding author: Dr Mohammadreza Vafa, rezavafa@yahoo.com DrMohammad Sharifzadeh, msharifzadeh@sina.tums.ac.ir

1. Department of Community Nutrition, School of Nutritional Science and Dietetics, Tehran University of Medical Sciences, Tehran, Iran.

2. Cellular and Molecular Research Center and Hematology Department, School of Allied Medical Science, Iran University of Medical Sciences, Tehran, Iran.

3. Department of Pharmacology, Pharmaceutical Science Research Center, Faculty of Pharmacy, Tehran University of Medical Sciences, Tehran, Iran.

4. Department of Cellular-Molecular Nutrition, School of Nutritional Science and Dietetics, Tehran University of Medical Sciences, Tehran, Iran.

5. Department of Pharmacology and Toxicology, Faculty of Pharmacy, Tehran University of Medical Sciences, Tehran, Iran.

6. Department of Nutrition, School of Public Health, Iran University of Medical Sciences, Tehran, Iran. and environmental factors affect its prevalence (Saxena et al. 2011). Observations have revealed that amyloid cascades, oxidative stress, neuroinflammation, insulin resistance, and apoptosis are involved in $\mathrm{AD}$ pathogenesis; nevertheless, the exact mechanism of $\mathrm{AD}$ is not known (Li et al. 2010).

Amyloid- $\beta$ (A $\beta) A \beta$ (1-42), produced by Amyloid protein precursor (APP), has a neurotoxicity effect and is

$\uparrow$ What is "already known" in this topic:

Studies have shown that probiotics can be a nutritional approach to improve metabolic markers such as FBS and lipid profile in patients with diabetes.

\section{$\rightarrow$ What this article adds:}

Disruption of insulin signaling cascade and insulin resistance can induce $\mathrm{AD}$ that causes systemic alterations in serum insulin, FBS levels, and lipid profile; however, probiotics can improve these markers. 
deposited in extracellular senile plaque composed of $\beta$ amyloid and following the aggregation of $A \beta$, microglial cells lose their protective function and cannot clear $A \beta$, which causes synaptic function loss, neurons apoptosis, oxidative stress, neuroinflammation, and memory loss (Butterfield et al. 2007, Cioanca et al. 2013, El Khoury and Luster. 2008). Since all these mechanisms are involved in pathogenesis of diabetes mellitus, many studies have shown that $\mathrm{AD}$ is associated with diabetes and $\mathrm{AD}$ is considered as Type 3 diabetes in which insulin resistance is the common cause of both diseases (Ríos et al. 2014). $\mathrm{A} \beta$ can compete with insulin or decrease insulin receptors binding affinity. It can also cause insulin signaling impairment and increase the susceptibility of neurons to oxidative stress and cause insulin resistance and disruption in memory (Kim and Feldman. 2015). Insulin signaling cascades have a neuroprotective role in the central nervous system (CNS) and improve synaptogenesis and synaptic remodeling against apoptosis and oxidative stress. Thus, disruption of insulin signaling cascade can induce AD. Central insulin resistance can lead to systemic alterations in insulin level (Burns et al. 2012) and other biomarkers including fasting blood sugar (FBS) and lipid profile (Schiffrin et al. 2010, Solfrizzi et al. 2006).

Many studies have found that using components such as nutrients or bioactive components of foods can improve oxidative stress and insulin resistance, and thus, they are useful and effective in improving pathological markers in AD (Hashimoto et al. 2009, Kumar et al. 2011, van der Beek and Kamphuis. 2008).

If probiotics, as a group of gastrointestinal microbiota bacteria, are consumed in adequate amounts, they can show useful effects in the host (Dinan and Cryan. 2012, Quigley. 2010). Although studies on microbiota and diversity of gut bacteria in those with Alzheimer's disease are limited, the results of one study have shown the increased gram-negative bacteria of microbiota in a transgenic rat model of Alzheimer's disease (Mayer et al. 2014). Observations have revealed that oxidative stress and inflammation in the Alzheimer's disease can lead to the increased gram-negative bacteria and the decreased gram-positive bacteria, or probiotics (Lye et al. 2009). Also, germ free mice showed cognitive dysfunction (Bhattacharjee and Lukiw. 2013). On the other hand, studies have demonstrated that aging as a risk factor of $\mathrm{AD}$ can cause a decrease in probiotics bacteria, especially in Bifidobacterium and Lactobacillus (Leung and Thuret. 2015, Marques et al. 2014) that have antiinflammatory and antioxidative properties (Ruan et al. 2015). Probiotics such as Lactobacillus and Bifidobacterium have beneficial effects on the metabolic profile and insulin resistance biomarkers (Shimizu et al. 2015). Moreover, specific species of lactic acid bacteria such as L.acidophilus improve glucose intolerance, hyperglycemia, and hyperinsulinemia (Ruan et al. 2015). Thus, the present study aimed at evaluating the probiotics effect on improving $A \beta$-induced insulin resistance in a rat model of Alzheimer's disease.

\section{Methods}

\section{Study Design}

In this study, 60 male Wistar rats (weight: 180-220 g, 8 weeks) were purchased from the Faculty of Pharmacy at Tehran University of Medical Sciences. Each rat was housed in one cage and all cages were kept at $25 \pm 2{ }^{\circ} \mathrm{C}$ temperature and on a 12- hour light/ dark cycle. All the rats had accessed to adlibitum food (chow) and water. Our experiments were approved by the ethical committee for the care and use of laboratory animals at Tehran University of Medical Sciences (code: 902-1324001) and council of graduated students of Iran University of Medical Sciences (code: 93-01-27-24403).

Rats were divided into 5 groups: (1) a control group (C) without surgical and dietary interventions; (2) a controlprobiotics (CP) group that received probiotics in drinking tap water for 8 weeks but received no surgical intervention; (3) a group receiving a sham operation (S) for which phosphate buffered saline (PBS), as the solvent of $\mathrm{A} \beta$, was injected intrahippocampus, but without any dietary intervention; (4) an Alzheimer group (A $\beta$ ) for which 50 $\mathrm{ng} / \mu \mathrm{L} /$ side $\beta$-amyloid was injected intrahippocampus, but without dietary intervention; and (5) an Alzheimerprobiotics (AP) group that received 2 g probiotics with drinking tap water during 8 weeks (with 4 weeks interval before and after $A \beta$ injection) in addition to surgical intervention ( $\mathrm{A} \beta$ injection).

\section{Beta- amyloid (1-42) preparation}

A $\beta$ (1-42) (sigma-Aldrich, USA) was dissolved in PBS (the solvent of $A \beta$ ). The solution was incubated for 5 days at $37^{\circ} \mathrm{C}$ and diluted by PBS to $50 \mathrm{ng} / \mu \mathrm{L}$ concentration on the test day. (A $\beta$ dosage was selected based on previous studies (Asadi et al. 2015, Eftekharzadeh et al. 2012, Shariatpanahi et al. 2015) and our pilot study).

\section{Probiotics preparation}

Powdered preparation probiotics was obtained from Tak-Gen Company (Tehran, Iran). Based on the results of our pilot study, water volume was calculated $(30 \mathrm{~mL})$ and $2 \mathrm{~g}$ of probiotics (probiotics dosage was selected based on a previous study (Davari et al. 2013) and our pilot study) including lactobacillus acidophilus, fermentum, Bifidobacterium lactis, and longum $\left(10^{10}\right.$ colony forming units $(\mathrm{CFU}) / \mathrm{g}$ ) were dissolved in an amount of calculated drinking tap water every morning. Water intake was monitored in all groups. Probiotics were selected based on antioxidative and anti-inflammatory effects, which have been reported in the past studies (Fijan. 2014, Hamaji et al. 2007, Messaoudi et al. 2011, Pang et al. 2012). Probiotics powder was colorless and odorless; at first, probiotics are inactive in water due to their special formulation, which includes a carrier compound that bonds to probiotics and allows probiotics activation in gut. Thus, the environmental factors do not affect probiotics in water.

\section{Surgery procedure}

Rats were anesthetized with an intraperitoneal injection of ketamine $(90 \mathrm{mg} / \mathrm{kg})$ and Xylazine $(5 \mathrm{mg} / \mathrm{kg})$ and were 
put in stereotaxic apparatus (Stoelting, wood Dale, IL, USA). For intrahippocampal injection of $\mathrm{A} \beta$, using atlas of Paxinos and Watson (anterior-posterior, $3.08 \mathrm{~mm}$; lateral, $\pm 2.2 \mathrm{~mm}$; dorsal-ventral $2.7 \mathrm{~mm}$ from bregma), CA1 areas of hippocampus were detected, and injection was performed using a Hamilton syringe $(50 \mathrm{ng} / \mu \mathrm{L} /$ side $)$. Every injection lasted for 1 minute; and to facilitate diffusion of $A \beta$, injection in the left side was performed after 60 seconds. Finally, the head of the rats was darned and they were returned to cages; probiotics intake began again in the next day.

\section{Glucose, Insulin levels, and Lipid profile detection}

After 12 hours of fasting, $5 \mathrm{~mL}$ blood was collected from all rats. The samples were kept for 30 minutes for clotting and were then centrifuged at $1500 \mathrm{rpm}$ for 10 minutes. Clear serum was collected and kept in -80 . Serum Glucose, triglyceride, total cholesterol, LDL, and HDL levels were detected by a calorimetric method with commercial kits (Dialab $\mathrm{GmbH}$, Austria). Insulin level was detected by ultrasensitive rat insulin ELISA kit (Mercodia, Sweden). Insulin resistance was determined using homeostasis model assessment of insulin resistance (HOMA-IR) using the following formula: fasting serum glucose $(\mathrm{mg} / \mathrm{dl}) \times$ fasting serum insulin $(\mathrm{MU} / \mathrm{dl}) / 405$.

\section{Statistical analysis}

Data were analyzed using SPSS 23.0. Results were presented as Mean \pm SD. ANOVA (one way analysis of variance followed Bonferroni comparisons post hoc) was used to compare all the variables between groups.

\section{Results \\ Weight changes}

At baseline and at the end of the study, weight was not significantly different between groups. During the intervention, only weight showed a significant difference on the third week in AP group compared to $\mathrm{C}, \mathrm{CP}$, and $\mathrm{S}$ groups (Fig. 1).

\section{Insulin resistance biomarkers}

Figure 2 illustrates serum FBS levels. A $\beta$ injection led to an increase in serum FBS level and indicated statistical difference in $\mathrm{A} \beta$ group $(156.83 \pm 19.39)$ compared to $\mathrm{C}$

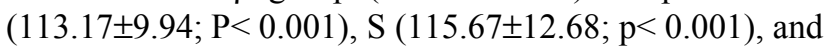
CP $(127.75 \pm 26.32 ; \mathrm{p}<0.001)$ groups. In the AP group,

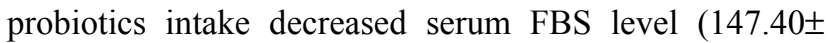
17.49) compared to $A \beta$ group, but it was not significant, but it was higher than $C, C P$, and $S(p<0.001)$ groups. $A \beta$ injection led to a statistically remarkable increase in serum insulin level (1.46 \pm 0.21$)$ (Fig. 3), and insulin resistance index was defined as HOMA-IR $(0.56 \pm 0.11)$ (Fig. 4) in $\mathrm{A} \beta$ group compared to $\mathrm{C}(0.78 \pm 0.32$ and $0.22 \pm 0.01), \mathrm{CP}$ $(0.79 \pm 0.29$ and $0.25 \pm 0.12)$, and $S(0.78 \pm 0.15$ and $0.22 \pm 0.05)$ groups $(\mathrm{p}<0.001)$. In AP group, probiotics intake considerably reduced serum insulin level $(0.93 \pm 0.14)$ and HOMA index $(0.33 \pm 0.05)$ compared to $\mathrm{A} \beta$ group $(\mathrm{p}<0.001)$.

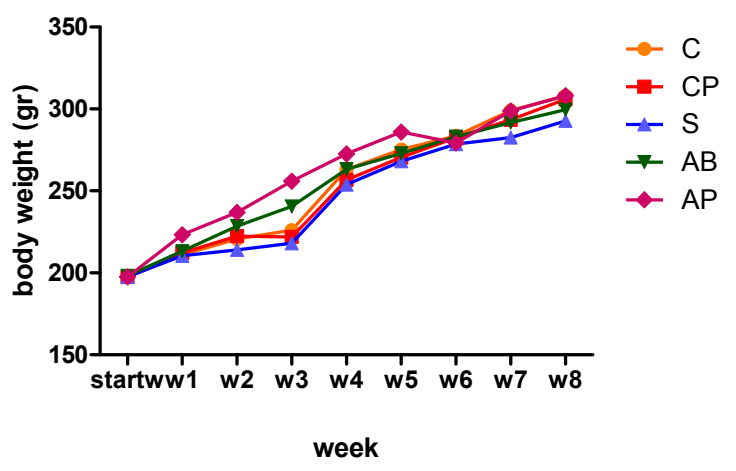

Fig. 1. The changes of body weight

At the baseline and at the end of study, weight did not show any significant difference between groups. During the intervention, except for difference in the third week, results did not show any statistical difference between the groups $(\mathrm{p}>0.05)$. $\mathrm{C}$ : control; $\mathrm{CP}$ : control-probiotics; S: sham operation; A $\beta$ : Alzheimer; AP: Alzheimer-probiotics.

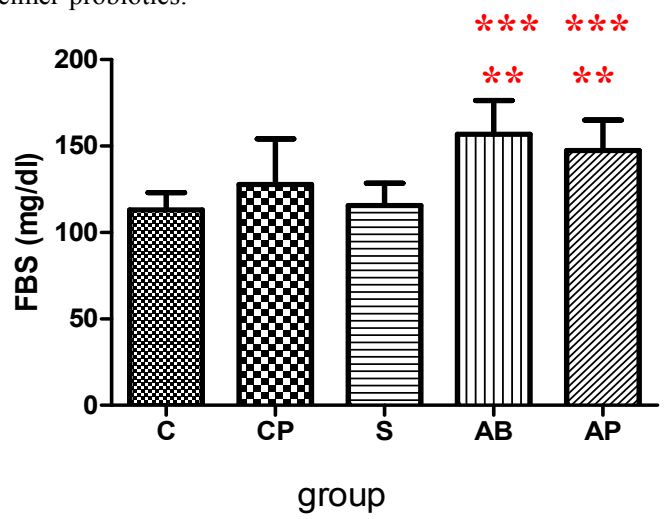

Fig. 2. The effect of intrahippocampal injection of $A \beta$ and $\mathrm{A} \beta+$ probiotics intake on FBS level

The results have been presented as Mean $\pm \mathrm{SD}$. $* * * \mathrm{P}<0.0001$ significant difference between $\mathrm{A} \beta$ vs $\mathrm{C}, \mathrm{A} \beta$ vs $\mathrm{S}$ and $\mathrm{AP}$ vs $\mathrm{C}$. $* * \mathrm{P}<0.01$ significant difference between $A \beta$ vs $C P$, AP vs $C P$ and $A P$ vs $S$. $\mathrm{C}$ : control; $\mathrm{CP}$ : control-probiotics; $\mathrm{S}$ : sham operation; $\mathrm{A} \beta$ : Alzheimer; AP: Alzheimer-probiotics.

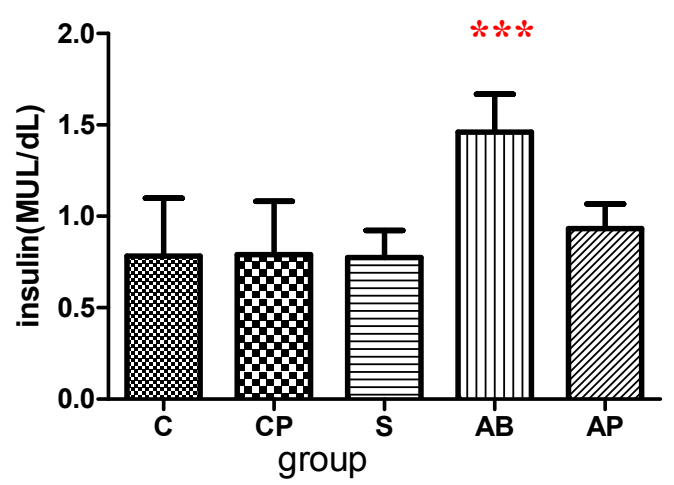

Fig. 3. The effect of intra hippocampal injection of $A \beta$ and $\mathrm{A} \beta+$ probiotics intake on insulin level

The results have been presented as Mean $\pm \mathrm{SD}$. $* * * \mathrm{P}<0.0001$ significant difference between $A \beta$ vs $C, A \beta$ vs $C P, A \beta$ vs $S$ and $A \beta$ vs AP. C: control; $C P$ : control-probiotics; $S$ : sham operation; $A \beta$ vs: Alzheimer; AP: Alzheimer-probiotics. 


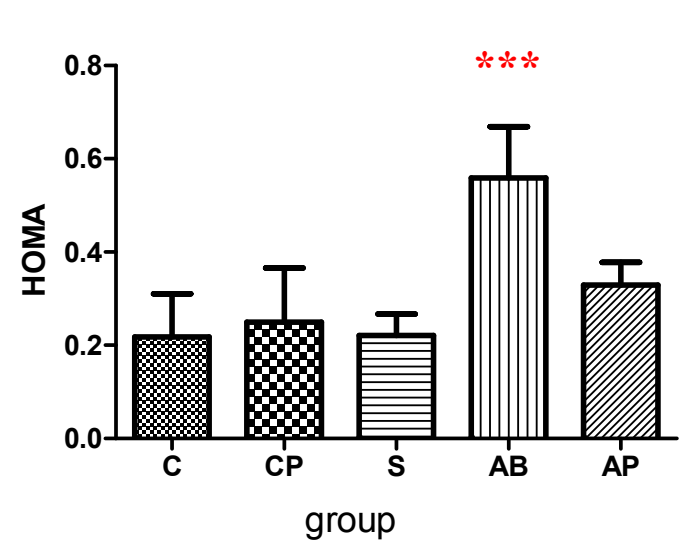

Fig. 4. The effect of intrahippocampal injection of $\mathrm{A} \beta$ and $\mathrm{A} \beta+$ probiotics intake on HOMA-IR index

The results have been presented as Mean \pm SD. $* * * \mathrm{P}<0.0001$ significant difference between $A \beta$ vs $C, A \beta$ vs $C P, A \beta$ vs $S$ and $A \beta$ vs $A P$. $\mathrm{C}$ : control; $\mathrm{CP}$ : control-probiotics; $\mathrm{S}$ : sham operation; $\mathrm{A} \beta$ : Alzheimer; AP: Alzheimer-probiotics.

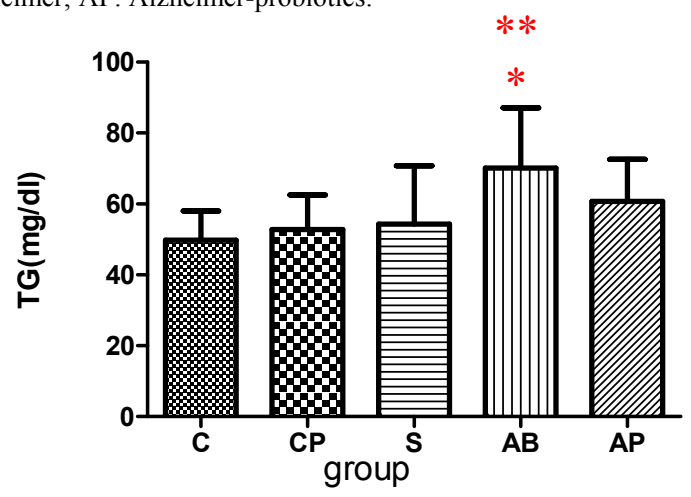

Fig. 5. The effect of intrahippocampal injection of $A \beta$ and $\mathrm{A} \beta+$ probiotics intake on TG level

The results have been presented as Mean $\pm \mathrm{SD}$. ${ }^{* *} \mathrm{P}<0.01$ significant difference between $A \beta$ vs $C$; $* \mathrm{P}<0.05$ significant difference between $A \beta$ vs $C P$ and $A \beta$ vs S. C: control; $C P$ : control-probiotics; $S$ : sham operation; A $\beta$ : Alzheimer; AP: Alzheimer-probiotics.

\section{Lipid profile}

According to lipid profile, $A \beta$ injection only increased serum TG level (Fig. 5) in A $\beta$ group (79.08 17.03 ) compared to $\mathrm{C}(49.83 \pm 8.21 ; \mathrm{p}<0.001), \mathrm{CP}(52.83 \pm 9.73)$ and $\mathrm{S}$ $(54.25 \pm 16.55)$ groups $(\mathrm{p}=0.03)$. In the AP group, probiotics intake reduced serum TG level $(60.70 \pm 11.92)$, but it was not significant compared to $\mathrm{A} \beta$ group and other groups. Total cholesterol, LDL, and HDL levels were not significantly different between groups (Table 1).

\section{Discussion}

In this study, we assessed insulin resistance biomarkers. Our results revealed higher serum FBS, insulin levels, and HOMA-IR index in the Alzheimer group compared to the control group. In the AP group, probiotics intake decreased FBS level, but it was not significant compared to the Alzheimer group; moreover, FBS level was higher than the control group. Nevertheless, fasting insulin level and HOMA-IR index considerably declined and returned to the optimal level in the AP group. With respect to lipid profile, only serum TG level dramatically increased in the Alzheimer group compared to the control, but TG level did not show any significant differences between the Alzheimer and AP groups.

Consistent with the results of our study, many studies have suggested increased level of serum glucose, insulin, and HOMA in AD patients without diabetes (Burns et al. 2012, Li et al. 2015, Qiu and Folstein. 2006). Some studies have indicated that insulin resistance index (HOMAIR) is mostly correlated with senile plaque and that $A D$ patients are highly likely to develop diabetes. For example, the results of one study indicated that $80 \%$ of $\mathrm{AD}$ patients suffer from Type 2 diabetes or an impaired fasting glucose level (Kim and Feldman. 2015). It is possible that increased peripheral insulin level suggested in $\mathrm{AD}$ is compensatory for decreased insulin level in CNS and insulin signaling impairment. Also, results of some studies have suggested that pathological alterations of the brain related to $\mathrm{AD}$ commonly happen in regions regulating metabolism and satiety and could lead to systemic alterations of insulin level (Burns et al. 2012). On the other hand, oxidative stress and inflammation observed in $\mathrm{AD}$ can affect systemic biomarkers. Various studies have found increased inflammatory and oxidative markers in the serum of AD patients. Inflammatory factors such as $\mathrm{TNF} \alpha$ lead to increased level of glucose and triglyceride production in the liver and decrease the peripheral glucose uptake, which causes insulin resistance (Mushtaq et al. 2015). Also, oxidative stress biomarkers can elevate FBS and HOMA-IR index (Salim et al. 2010). The elevated glucose level can affect the glucose uptake and may increase denevo lipogenesis and TG synthesis (Yadav et al. 2007).

Probiotics such as lactobacillus and bifidobacterium have beneficial effects on metabolic profile (Shimizu et al. 2015). Specific species of lactic acid bacteria such as L.acidophilus improve glucose intolerance, hyperglycemia, and hyperinsulinemia (Ruan et al. 2015). One Study shows that consumption of capsules containing L.acidophillus, L.casei, and B.bifidum decreases serum

Table 1. The serum lipid profile biomarkers

\begin{tabular}{lccc} 
Table 1. The serum lipid profile biomarkers & & TDL \\
Group & $\begin{array}{c}\text { Total Cholesterol } \\
(\mathrm{mg} / \mathrm{dl})\end{array}$ & $\begin{array}{c}\text { HDL } \\
(\mathrm{mg} / \mathrm{dl})\end{array}$ & $27.08 \pm 3.89$ \\
\hline Control & $68.5 \pm 9.85$ & $34.17 \pm 5.81$ & $27.08 \pm 3.92$ \\
CP & $75.33 \pm 10.56$ & $35.42 \pm 5.29$ & $25.75 \pm 4.20$ \\
S & $67.25 \pm 8.58$ & $33.92 \pm 5.5$ & $26.08 \pm 3.96$ \\
A & $69.25 \pm 9.21$ & $32.67 \pm 5.23$ & $25.00 \pm 3.05$ \\
AP & $66.70 \pm 6.5$ & $30.10 \pm 2.88$ & \\
\hline
\end{tabular}

The results are in Mean $+\mathrm{SD}$

There was no significant difference between the groups $(\mathrm{P}>0.05)$. CP: control-probiotics, $\mathrm{S}$ : sham operation; A $\beta$ : Alzheimer; AP: Alzheimer-probiotics. 
insulin level and HOMA-IR, but it does not show any effect on FBS level and lipid profile in patients with major depressive disorder (Akkasheh et al. 2016). Another study suggests that intake of probiotics including L.acidophillus, L.fermentum, and B.lactis have resulted in a decreased level of FBS and insulin in STZ-induced diabetic rats (Davari et al. 2013).The results of a meta-analysis suggest that decreased level of glucose following probiotics intake is significantly higher in hyperglycemic objects. Interventions with duration over 8 weeks reveal a remarkable effect on FBS level; however, in interventions with durations of less than or equal to 8 weeks, a slight trend of glucose reduction have been observed. Also, amount of bacteria $\geq 10^{11} \mathrm{CFU}$ is related to significant effects (Desbonnet et al. 2010). So, alteration in FBS, insulin levels, and HOMA-IR are confirmed in our study.

Also, probiotics cause PPAR $\alpha$ (peroxisome proliferatoractivated receptor) and CPT2 (carnitine-palmitoyltransferase) expression up-regulation, $\beta$-oxidation activation, and lipogenesis suppression that eventually lead to decreased TG level, as observed in our study (Park et al. 2013). Another meta-analysis demonstrated that probiotics significantly reduce the total cholesterol and LDL levels and they do not have any effect on HDL or TG levels. It is noted that when the baseline cholesterol and LDL levels are optimal, probiotics do not show any effects. The hypocholesterolemic effect of probiotics is stronger in trials over than or equal to 8 weeks. Also, bacterial strains and dosage are important factors (Cho and Kim. 2015). Since there were fewer baseline levels of cholesterol and LDL in our study, these parameters did not change.

Nevertheless, there were some limitations in our study. Given the costs associated with such undertaking, it was not possible to prolong the study. Also, it was better to study genetically modified mice as $\mathrm{AD}$ phenotype to avoid additional inconsistencies.

\section{Conclusion}

Overall, our study results indicate that probiotics can decrease insulin resistance markers such as serum insulin levels and HOMA-index, which have raised following intrahippocampal $A \beta$ injection in an experimental model of Alzheimer's disease.

\section{Acknowledgements}

This study was a part of a Ph.D. thesis financially supported by Deputy of Research, Iran University of Medical Sciences (Grant No. 93-01-27-24403). Also, the authors declare no conflicts of interest.

\section{Conflict of Interests}

The authors declare that they have no competing interests.

\section{References}

1. Tillisch K, Labus J, Kilpatrick L, Jiang Z, Stains J, Ebrat B., et al. Consumption of fermented milk product with probiotic modulates brain activity. Gastroenterol. 2013;144(1394-1401.e4): 10.1053/j.gastro.2013.02.043
2. Ríos $\mathrm{J}$ A, Cisternas $\mathrm{P}$, Arrese $\mathrm{M}$, Barja $\mathrm{S}$, Inestrosa NC. Is Alzheimer's disease related to metabolic syndrome? A Wnt signaling conundrum. Prog Neurobiol. 2014;121 (125-146): 10.1016/j.pneurobio.2014.07.004

3. Saxena A K, Majeed A, Saad S, Oothuman P, Mahmoud M, AlDeen M I. Lipid peroxidation in chronic cerebral hypoperfusioninduced neurodegeneration in rats. Inter Med J Malay. 2011;10 (36):

4. Li J, Wang C, Zhang J H, Cai J-M, Cao Y-P, Sun X-J. Hydrogenrich saline improves memory function in a rat model of amyloidbeta-induced Alzheimer's disease by reduction of oxidative stress. Brain Res. 2010;1328 (152-161): 10.1016/j.brainres.2010.02.046

5. Butterfield D A, Reed T, Newman S F, Sultana R. Roles of amyloid $\beta$-peptide-associated oxidative stress and brain protein modifications in the pathogenesis of Alzheimer's disease and mild cognitive impairment. Free Radic Biol Med. 2007;43 (658-677): 10.1016/j.freeradbiomed.2007.05.037

6. Cioanca O, Hritcu L, Mihasan M, Hancianu M. Cognitive-enhancing and antioxidant activities of inhaled coriander volatile oil in amyloid $\beta(1-42)$ rat model of Alzheimer's disease. Physiol Behav. 2013;120 (193-202): 10.1016/j.physbeh.2013.08.006

7. El Khoury J, Luster AD. Mechanisms of microglia accumulation in Alzheimer's disease: therapeutic implications. Trends Pharmacol Sci. 2008;29 (626-632): 10.1016/j.tips.2008.08.004

8. Kim B, Feldman EL. Insulin resistance as a key link for the increased risk of cognitive impairment in the metabolic syndrome. Experimental Molecul Med. 2015; 47 (e149): 10.1038/emm.2015.3

9. Burns JM, Honea RA, Vidoni ED, Hutfles LJ, Brooks WM, Swerdlow RH. Insulin is differentially related to cognitive decline and atrophy in Alzheimer's disease and aging. Biochimica Biophysica Acta (BBA)-Molecular Basis Dis. 2012;1822 (333-339): 10.1016/j.bbadis.2011.06.011

10. Schiffrin EJ, Morley JE, Donnet-Hughes A, Guigoz Y. The inflammatory status of the elderly: the intestinal contribution. Mutat Res Fundament Molcul Mechanism Mutagen. 2010; 690 (50-56): 10.1016/j.mrfmmm.2009.07.011

11. Solfrizzi V, D'introno A, Colacicco A M, Capurso C, Todarello O, Pellicani V., et al. Circulating biomarkers of cognitive decline and dementia. Clinica Chimica Acta. 2006;364 (91-112): 10.1016/j.cca.2005.06.015

12. Hashimoto M, Hossain S, Tanabe Y, Kawashima A, Harada T, Yano $\mathrm{T}$, et al. The protective effect of dietary eicosapentaenoic acid against impairment of spatial cognition learning ability in rats infused with amyloid $\beta(1-40)$. J Nutr Biochem. 2009;20 (965-973): 10.1016/j.jnutbio.2008.08.009

13. Kumar M, Verma V, Nagpal R, Kumar A, Gautam S K, Behare P $\mathrm{V}$., et al. Effect of probiotic fermented milk and chlorophyllin on gene expressions and genotoxicity during AFB 1-induced hepatocellular carcinoma. Gene. 2011; 490 (54-59): 10.1016/j.gene.2011.09.003

14. Van Der Beek EM, Kamphuis PJ. The potential role of nutritional components in the management of Alzheimer's Disease. Eur J Pharmacol.2008;585 (197-207): 10.1016/j.ejphar.2008.01.049

15. Dinan TG, Cryan JF. Regulation of the stress response by the gut microbiota: implications for psychoneuroendocrinology Psychoneuroendocrino. 2012;37 (1369-1378): 10.1016/j.psyneuen. 2012.03.007

16. Quigley EM. Prebiotics and probiotics; modifying and mining the microbiota. Pharmacol Res. 2010; 61 (213-218): 10.1016/j.phrs.2010.01.004

17. Mayer E A, Knight R, Mazmanian S K, Cryan J F, Tillisch K. Gut microbes and the brain: paradigm shift in neuroscience. $\mathrm{J}$ Neuroscience.2014; 34 (15490-15496).

18. Lye H-S, Kuan C-Y, Ewe J-A, Fung W-Y, Liong M-T. The improvement of hypertension by probiotics: effects on cholesterol, diabetes, renin, and phytoestrogens. Inter J Mol Sci. 2009;10 (37553775): 10.3390/ijms10093755

19. Bhattacharjee S, Lukiw W J. Alzheimer's disease and the microbiome. Front Cell Neurosci.2013;7(1-4): 10.3389/fncel.2013. 00153

20. Leung K, Thuret S. Gut Microbiota: A Modulator of Brain Plasticity and Cognitive Function in Ageing. Healthcare. 2015. Multidisciplinary Digital Publishing Institute.

21. Marques TM, Cryan JF, Shanahan F, Fitzgerald GF, Ross RP, Dinan TG., et al. Gut microbiota modulation and implications for host 
health: dietary strategies to influence the gut-brain axis. Innov Food Sci Emerg. 2014;22 (239-247):10.1016/j.ifset.2013.10.016

22. Ruan Y, Sun J, He J, Chen F, Chen R, Chen H. Effect of Probiotics on Glycemic Control: A Systematic Review and Meta-Analysis of Randomized, Controlled Trials. PloSone. 2015;10 (e0132121): 10.1371/journal.pone.0132121

23. Shimizu M, Hashiguchi M, Shiga T, Tamura H-O, Mochizuki M. Meta-Analysis: Effects of Probiotic Supplementation on Lipid Profiles in Normal to Mildly Hypercholesterolemic Individuals. PloSone. 2015;10 (e0139795): DOI:10.1371/journal.pone.0139795

24. Asadi F, Jamshidi A H, Khodagholi F, Yans A, Azimi L, Faizi M., et al. Reversal effects of crocin on amyloid $\beta$-induced memory deficit: Modification of autophagy or apoptosis markers. Pharmacol Biochem Be. 2015;139 (47-58): 10.1016/j.pbb.2015.10.011

25. Eftekharzadeh B, Ramin M, Khodagholi F, Moradi S, Tabrizian K, Sharif R., et al. Inhibition of PKA attenuates memory deficits induced by $\beta$-amyloid $(1-42)$, and decreases oxidative stress and NF- $\kappa \mathrm{B}$ transcription factors. Behav brain Res. 2012;226 (301-308): 10.1016/j.bbr.2011.08.015

26. Shariatpanahi M, Khodagholi F, Ashabi G, Khasraghi A A, Azimi L, Abdollahi M., et al. Ameliorating of Memory Impairment and Apoptosis in Amyloid $\beta$-Injected Rats Via Inhibition of Nitric Oxide Synthase: Possible Participation of Autophagy. IJPR. 2015. 14 (811):

27. Davari S, Talaei S, Alaei H. Probiotics treatment improves diabetesinduced impairment of synaptic activity and cognitive function: behavioral and electrophysiological proofs for microbiome-gutbrain axis. Neuroscience. 2013; 240 (287-296): $/ 10.1016 /$ j.neuroscience.2013.02.055

28. Fijan S. Microorganisms with claimed probiotic properties: an overview of recent literature. Int J Environ Res Pub Health. 2014;11 (4745-4767): 10.3390/ijerph110504745

29. Hamaji Y, Fujimori M, Sasaki T, Matsuhashi H, Matsui-Seki K, Shimatani-Shibata Y., et al. Strong enhancement of recombinant cytosine deaminase activity in Bifidobacterium longum for tumortargeting enzyme/prodrug therapy. Biosci Biotechnol Biochem. 2007;71 (874-883): 10.1271/bbb.60502

30. Messaoudi M, Lalonde R, Violle N, Javelot H, Desor D, Nejdi A, et al. Assessment of psychotropic-like properties of a probiotic formulation (Lactobacillus helveticus R0052 and Bifidobacterium longum R0175) in rats and human subjects. Br J Nutr. 2011;105 (755-764): 10.1017/S0007114510004319

31. Pang G, Xie J, Chen Q, Hu Z. How functional foods play critical roles in human health. Food Sci Human Well. 2012;1 (26-60): 10.1016/j.fshw.2012.10.001

32. Li X, Song D, Leng SX. Link between type 2 diabetes and Alzheimer's disease: from epidemiology to mechanism and treatment. Clinical Interven Aaging. 2015;10 (549): dx.doi.org/10.2147/CIA.S74042

33. Qiu WQ, Folstein MF. Insulin, insulin-degrading enzyme and amyloid- $\beta$ peptide in Alzheimer's disease: review and hypothesis. Neurobiol Aging. 2006;27 (190-198): :10.1016/j.neurobiolaging. 2005.01.004

34. Mushtaq G, Khan JA, Kumosani TA, Kamal MA. Alzheimer's disease and type 2 diabetes via chronic inflammatory mechanisms. Saudi J Biolog Sie. 2015.22 (4-13): /dx.doi.org/10.1016/j.sjbs. 2014.05.003

35. Salim S, Asghar M, Chugh G, Taneja M, Xia Z, Saha K. Oxidative stress: a potential recipe for anxiety, hypertension and insulin resistance. Brain Res. 2010;1359 (178-185): 10.1016/j.brainres. 2010.08.093

36. Yadav H, Jain S, Sinha P. Antidiabetic effect of probiotic dahi containing Lactobacillus acidophilus and Lactobacillus casei in high fructose fed rats. Nutri Metabol Cardiovascul Dis. 2007.23 (62-68):

37. Akkasheh G, Kashani-Poor Z, Tajabadi-Ebrahimi M, Jafari P, Akbari H, Taghizadeh M., et al. Clinical and metabolic response to probiotic administration in patients with major depressive disorder: A randomized, double-blind, placebo-controlled trial. Nutri Metabol Cardiovascul Dis..2016;32 (315-320): 10.1016/j.nut.2015.09.003

38. Desbonnet L, Garrett L, Clarke G, Kiely B, Cryan J , Dinan T. Effects of the probiotic Bifidobacterium infantis in the maternal separation model of depression. Neuroscience. 2010;170 (11791188): : 10.1016/j.neuroscience.2010.08.005

39. Park D-Y, Ahn Y-T, Huh C-S, Mcgregor RA, Choi M-S. Dual probiotic strains suppress high fructose-induced metabolic syndrome. World J Gastroenterol. 2013;19 (274-283): 10.3748/wjg.v19.i2.274

40. Cho Y A, Kim J. Effect of Probiotics on Blood Lipid Concentrations: A Meta-Analysis of Randomized Controlled Trials. $\begin{array}{llll}\text { Med Conflict Survival. } 2015.94 & \text { (e1714): } 10.1097 / \mathrm{MD} \text {. }\end{array}$ 0000000000001714 